\title{
The role of etravirine in the management of treatment-experienced pediatric patients with HIV
}

This article was published in the following Dove Press journal:

HIVIAIDS - Research and Palliative Care

9 April 2013

Number of times this article has been viewed

\section{Danielle Osterholzer}

Ryan White Center for Pediatric Infectious Diseases, Riley Hospital for Children at Indiana University Health, Division of Adult Infectious Diseases, Indiana University School of Medicine, Indianapolis, IN, USA
Correspondence: Danielle Osterholzer Indiana University, Infectious Diseases, 545 Barnhill Drive, Emerson Hall 42I, Indianapolis, IN 46202, USA

$\mathrm{Tel}+\mathrm{I} 3172747936$

Fax + I 317274 |587

Email dosterho@iu.edu
Abstract: Pediatric patients infected with human immunodeficiency virus (HIV) are now living longer, healthier lives due to the advent of combined antiretroviral (ARV) therapy, including regimens that often contain non-nucleoside reverse transcriptase inhibitors (NNRTIs). However, first-generation NNRTIs such as nevirapine (NVP) and efavirenz (EFV) have a low genetic barrier to resistance, and both drugs can become ineffective with a single viral point mutation. New agents with activity against resistant viral strains must be available to salvage children and adolescents with virologic failure after NNRTI use. One such drug, etravirine, an oral secondgeneration NNRTI approved for use in the US in heavily treatment-experienced HIV-1-infected adults in 2008, is accumulating data in this younger population. Etravirine became approved by the US Food and Drug Administration in early 2012 to be used in combination with other ARV medications in HIV-1-infected children aged 6 years to $<18$ years who are failing their regimens with HIV-1 strains resistant to NNRTIs and other ARVs. This approval was largely based on data from a prospective, open-label, phase II clinical trial in this age group prescribed etravirine at $5.2 \mathrm{mg} / \mathrm{kg}$ twice daily (up to the adult dose of $200 \mathrm{mg}$ twice daily) in combination with an investigator-selected optimized background regimen. Currently available 48-week follow-up data show complete viral suppression $(<50$ copies $/ \mathrm{mL})$ in $56 \%$ of the patients, with relatively few serious adverse events attributed to the drug. Additional studies and case reports from the field suggest its utility in clinical practice. This review is designed to increase the background understanding of this drug in pediatric HIV providers, to lay out the current pediatric data to support its use, and to define its practical role in the treatment of HIV-infected children now and in the future.

Keywords: salvage, resistance, children, adolescents, NNRTI, perinatal

\section{Introduction}

In the early years of the human immunodeficiency virus (HIV) epidemic, infants and children infected with HIV had relatively little hope of survival into adolescence, because there were no effective therapies to halt the virus. ${ }^{1,2}$ Now, 30 years later, HIV-infected children and adolescents can live much longer, some well into adulthood, provided they are treated early and are compliant with appropriate combination antiretroviral (ARV) therapy (cART). ${ }^{3}$ The US Department of Health and Human Services treatment guidelines recommend that all HIV-infected children aged $<12$ months be treated with cART regardless of baseline CD4 percentage or viral load (VL) measurement. ${ }^{4}$ Once started, these regimens are usually continued for the life of the patient. Currently, over 20 distinct ARV compounds from six different classes are approved for use in children or adolescents with HIV infection in the US. ${ }^{4}$ However, effective cART regimens usually 
require three or more active drugs from at least two different classes. With the currently available ARVs, the number of combinations that can be devised to fully suppress the virus in pediatric patients is finite. In addition, now that children are living longer, they need sequential regimens that will be active and overcome prior accumulated resistance for many decades, while promoting adherence and minimizing toxicity. Therefore, new ARVs must continue to be developed, studied, and approved in children that can salvage prior virologic failures (VFs). Etravirine (ETR), a second-generation nonnucleoside reverse transcriptase inhibitor (NNRTI), was approved in 2008 for heavily treatment-experienced adults with multiclass ARV resistance after randomized, placebocontrolled clinical trials (DUET-1 and DUET-2) showed its superiority when combined with an optimized background regimen in salvaging adults with triple-class resistance versus placebo $(61 \%$ versus $40 \%$ with VL $<50$ copies $/ \mathrm{mL}) .{ }^{5-7}$ To be included in these studies, all patients had to have at least one NNRTI resistance mutation at baseline. Recently, based on the results of phase I and II clinical trials in children, ETR has now been approved for use in children aged 6 years to $<18$ years. ${ }^{8}$ The role of this drug in pediatric HIV patients will be discussed.

\section{Resistance in pediatric HIV infection}

Children with HIV infection carry with them a whole host of innate and external factors that can contribute to the development of HIV drug resistance mutations. First of all, infants who are perinatally infected with HIV often have extremely high VLs initially, which take longer to suppress than those in older children or adults, even when on fully active cART regimens. ${ }^{9-11}$ The pharmacokinetics of many ARVs in the setting of prematurity and early infancy are unknown or unpredictable, making appropriate dosing of these drugs difficult at times. These factors can lead to subtherapeutic ARV levels. One can speculate that active viral replication in this setting predisposes to the development of viral mutants that confer resistance to the drugs being given, especially to those with traditionally low barriers to resistance, such as nevirapine (NVP). In addition, given that the rate of transmitted phenotypic ARV resistance acquired in ARV-naïve adults is estimated to be $>12 \%$, mothers with HIV are increasingly likely to infect their infants with HIV strains resistant to certain ARVs, including NNRTIs such as NVP and efavirenz (EFV). ${ }^{12-14}$ Adolescents who become infected with HIV behaviorally are also at risk of acquiring transmitted NNRTI resistance. Lastly, in many resource-limited settings, single-dose NVP is given to infants at the time of delivery in an attempt to prevent mother to child transmission of HIV. Studies have shown that these infants, if they ultimately become infected, do not respond as well to NNRTI-based regimens as children not exposed perinatally to NVP. ${ }^{15,16}$ This is likely due to the long half-life of the drug and ongoing exposure of the virus to low drug levels during a time of rapid replication, which leads to resistance mutations. In addition to these innate factors, children have many external factors contributing to the development of resistance. HIVinfected children are likely to live in households where one or more parent may be suffering from HIV themselves, impairing the caregiver's ability to be compliant with the child's medication regimen. In addition, caregiver adherence to the prescribed therapy may be suboptimal, given the complexities of infant and child regimens. Often, children are living in difficult home situations, which can lead to chaotic environments where dispensing medications is not the first priority. In addition, some liquid formulations of ARVs have poor palatability, leading children to refuse medications. ${ }^{17}$ Also, as children age, they may not be informed of their diagnosis of HIV infection and may not appreciate the reason they are taking medication, leading to poor compliance. ${ }^{18}$ In the past, due to lags in the study and development of ARVs targeted for children, use of new drugs in this age group has lagged behind that in adults. Consequently, many children infected with HIV in the late 1990s, who are adolescents today, may have been maintained on single, dual, or other nonsuppressive regimens for years at a time, only to accumulate enormous numbers of resistance mutations to the commonly used classes of ARVs. ${ }^{19} \mathrm{NVP}$ and EFV have been the mainstay of cART in children when prescribed with a two-drug nucleoside reverse transcriptase inhibitor (NRTI) backbone, especially in developing countries. However, the first-generation NNRTIs have a low genetic barrier to resistance, such that upon VF, two signature point mutations, Y181C (NVP) and K103N (EFV), often develop, which have traditionally rendered the entire class of drugs useless. ${ }^{20}$ ETR has a much higher threshold for the development of resistance than NVP or EFV, requiring three or more of a certain group of ETR resistance-associated mutations (RAMs) to lose efficacy. ${ }^{21}$ In addition, the signature EFV point mutation, the $\mathrm{K} 103 \mathrm{~N}$, seems to have little, if any, effect on the success of ETR in adults with this mutation. ${ }^{7,22}$ These factors make ETR a possible tool in successful treatment of the NNRTI-experienced child.

\section{Etravirine clinical trial data in children}

Based on the success of the drug in multiclass, experienced, NNRTI-resistant adults, a phase I, open-label, 
two-stage, nonrandomized clinical trial was conducted in treatment-experienced HIV-1-infected children and adolescents between the ages of 6 years and $<18$ years to determine a weight-based dose of ETR that achieved comparable exposures with those of adults treated successfully with the drug. The clinical trial also assessed the short-term safety and tolerability of the drug. ${ }^{23}$ Children were recruited who were on stable lopinavir/ritonavir (LOP/r)-based ARV regimens plus two NRTIs plus or minus enfuvirtide (T-20), in addition to the study drug, ETR, for 7 days. No investigational drugs in children other than tenofovir were allowed. HIV VLs had to be undetectable or $<50$ copies/mL at baseline. Twenty patients completed stage I (ETR dosed at $4 \mathrm{mg} / \mathrm{kg}$ twice daily) and 21 patients completed stage II (ETR dosed at $5.2 \mathrm{mg} / \mathrm{kg}$ twice daily). Pharmacokinetic results indicated that stage II dosing was preferable, as it was less likely to lead to subtherapeutic ETR levels. The level of virus remained undetectable at the end of the study period in all children. In addition, there were no deaths or serious adverse events (AEs) attributable to the study drug during this trial. ${ }^{23}$

Subsequently, a 48-week, phase II, open-label, single-arm trial entitled the Pediatric Trial with Intelence ${ }^{\circledR}$ (Etravirine) as an Active NNRTI Option (PIANO) was designed to assess the efficacy, safety, and pharmacokinetics of ETR $5.2 \mathrm{mg} / \mathrm{kg}$ (max dose $200 \mathrm{mg}$ ) twice daily in HIV-1-infected, treatmentexperienced children (aged 6 years to $<12$ years) and adolescents (aged 12 years to $<18$ years) with VL $\geq 500$ copies $/ \mathrm{mL}$. Children with evidence of resistance to ETR on pretrial resistance testing were excluded. In addition to ETR, each patient received an investigator-selected optimized background regimen consisting of a ritonavir-boosted protease inhibitor (PI), plus NRTIs, and optional T-20 and/or raltegravir (RAL). This trial was completed in August 2011; however, data are available only in the form of abstracts at meetings or on the National Institutes of Health clinical trials website (Identifier NCT00665847). ${ }^{24,25}$ Overall, 101 patients were enrolled (60 adolescents and 41 children) of which $63 \%$ were female and about half were Caucasian. At baseline, the median VL was $3.9 \log _{10}$ copies $/ \mathrm{mL}$ (range $2-7 \log _{10}$ copies $/ \mathrm{mL}$ ) and CD4 count was 385 cells $/ \mathrm{mm}^{3}$ (range $7-1441$ cells $/ \mathrm{mm}^{3}$ ). Of the 101 subjects, $9 \%$ had used two NNRTIs in the past, whereas $66 \%$ has used one. Interestingly, 25\% (25) of the patients had never used an NNRTI before the trial, including $34 \%$ (14) of the children. In terms of efficacy, $68 \%$ (28) of the children and $48 \%$ (29) of the adolescents had week 48 HIV-1 $\mathrm{VL}<50$ copies $/ \mathrm{mL}$, where noncompleters equaled failure. The overall rate of suppression in study subjects at week 48 was $56 \%$. Forty-one $(41 \%)$ of the patients were classified as VF, where 29 did not respond at all and twelve had viral rebound after initial response. Of the $30 \mathrm{VFs}$ with genotypic data available at the end of the study, 18 (60\%) developed NNRTI RAMs, most commonly the Y181C ( $\mathrm{n}=8)$, E138A $(n=3)$, L100I $(n=3)$, and/or the V90I $(n=3)$. There was a significant dropout rate of $25 \%$ (25 patients), of which the authors state $8 \%$ was due to noncompliance and $8 \%$ due to AEs. Only $39 \%$ of the subjects were $>95 \%$ adherent by pill count, and $70 \%$ were $>80 \%$ adherent, indicating a large amount of noncompliance. The most common drug-related $\mathrm{AE}$ was rash in $18 \%$, but only $4 \%$ discontinued the drug for this reason. Diarrhea was second at $7 \%$. The rate of serious AEs was 5\%. The authors concluded that the safety, efficacy, and resistance development in this trial was similar to that seen in the adult DUET $^{7}$ trials, and responses were better in children over adolescents, most likely because of less previous NNRTI use and better adherence to their ARVs. ${ }^{24,25}$ Largely, due to this phase II trial, ETR was approved by the US Food and Drug Administration in early 2012 for use with other ARVs in treatment-experienced children aged 6 years to $<18$ years weighing at least $16 \mathrm{~kg}$ experiencing VF with virus resistant to NNRTIs. ${ }^{26} \mathrm{~A}$ phase III trial is currently recruiting participants in this age group to continue the provision of drug to pediatric patients previously enrolled in this clinical trial who are benefiting from ETR but who cannot access the drug through another source. This continuation study is expected to be complete in December 2013 and should give longer-term follow-up data on the use of ETR in this group. ${ }^{27}$ In addition, a similar phase I/II open-label trial to evaluate the safety, tolerability, pharmacokinetics, and antiviral activity of ETR in ARV-experienced children aged $>2$ months to $<6$ years is planned but not yet recruiting participants (NCT01504841). ${ }^{28}$

One additional prospective clinical trial that is still ongoing in Thailand gives some additional data for the use of ETR in third-line salvage regimens in children. This study is an observational study enrolling HIV-infected children aged 2 years to 18 years who require a regimen of darunavir/ ritonavir (DRV/r), ETR, or RAL for triple-class failure or toxicity to the PI in the second-line regimen (NCT01225406). Results are available in the form of an abstract from the International AIDS Conference in July 2012. ${ }^{29}$ Forty-four children were enrolled, 35 due to triple-class failure (NRTI, NNRTI, and PI) and nine due to hyperlipidemia to the PI in their second-line regimen. There was a high rate of NNRTI resistance, with $74 \%$ having at least one NNRTI mutation and $29 \%$ harboring $\geq 2.5$ ETR RAMs. The third-line regimen contained, on average, 4.5 drugs, with almost all containing 
$\mathrm{DRV} / \mathrm{r}$ and 30/44 containing ETR. Although they did not separate the results for those children receiving ETR from those not receiving the drug, after a mean of 6 months of therapy, $70 \%$ of the 44 children had VLs $<50$ copies $/ \mathrm{mL}$ compared with $20 \%$ at baseline. It is unclear whether those who failed did so because of noncompliance versus resistance to the third-line regimen, but the authors suspected that lack of adherence was largely to blame.

\section{Other studies of etravirine use in clinical practice}

Prior to ETR's approval in the US, a few groups from several countries have reported their limited experience using ETR in pediatric patients. Most of these reports outline the use of the drug in multidrug-resistant adolescents. One French paper by Thuret et $\mathrm{al}^{30}$ describes their use of ETR in combination with RAL and DRV/r in twelve heavily ARV-treated, NNRTI-resistant, perinatally infected adolescents with VF as part of an expanded access program. Over $10 \%$ of the patients had ETR resistance based on genotype testing and interpretation prior to initiation on this salvage regimen. The median CD4 count was 124 cells $/ \mathrm{mm}^{3}$ at baseline, indicating the advanced nature of their illness. All twelve patients had VL measurements $<400$ copies/mL by month 9 of treatment, whereas five of twelve had $<50$ copies/mL by the same time point. The median CD4 count dramatically increased to 500 cells $/ \mathrm{mm}^{3}$ by month 9 . At the last follow-up evaluation, only one patient had VF (two consecutive VLs $>400$ copies $/ \mathrm{mL}$ ) and had undetectable levels of ARVs in serum, indicating noncompliance. ${ }^{30}$ Another multicenter, retrospective study in Spain of 23 multidrug-resistant, pediatric patients (five children aged 5-12 years and 18 adolescents aged 13-18 years) published in 2011 showed that despite $70 \%$ of the patients harboring one or more ETR RAMs, after ETR-based therapy as part of a compassionate use program, 20 patients $(87 \%)$ achieved VL $<400$ copies/mL and 18 achieved VL $<50$ copies $/ \mathrm{mL} .{ }^{31}$ All children in this study had baseline NNRTI resistance except one NNRTI-naïve adolescent. Almost all of the patients $(91 \%)$ had at least two active ARVs in their regimen. However, three patients who also received DRV/r in addition to ETR did not achieve undetectable virus levels. All three had poor adherence. ETR-based therapy was replaced in three patients who had virologic and immunologic failure. Those patients had ETR RAMs at baseline (Y181C, G190A, and K101E plus G190A/S, respectively). In this study, AEs attributed to ETR were minimal and did not require drug discontinuation. ${ }^{31}$ Although most patients did well, poor compliance and baseline ETR resistance may have contributed to failure in some patients. Although most of the aforementioned studies involved older children and adolescents, one case report from Italy highlights the successful salvage of a perinatally infected toddler at age 2 years who harbored vertically acquired multidrug-resistant virus with a regimen of ETR, DRV/r, and lamivudine. ${ }^{32}$ The child's VL became undetectable ( $<50$ copies/mL) and CD4 percentage rose from $<5 \%$ to $>30 \%$ between 12 months and 15 months. Of note, at baseline, the child was fortunate enough to have only one DRV/r-associated mutation, and the NNRTI resistance mutations the child possessed (A98S, V118I, V179E, and Y188L) would be predicted to have little to no effect on the susceptibility of ETR based on the 2011 International AIDS Society Drug Resistance Mutations Guideline. ${ }^{33}$ The high degree of activity of these two drugs was largely responsible for his dramatic response.

\section{Limitations of etravirine}

As demonstrated earlier, although it is clear from clinical trial data that ETR has a higher barrier to resistance than its firstgeneration counterparts EFV and NVP, and has clearly contributed to regimen salvage in pediatric patients with NNRTI resistance, it is still unclear how much ETR has been responsible for the success versus the fact that it has been combined with other, newer, potent ARVs such as DRV/r, RAL, or the fusion inhibitor T-20, and whether it is the synergistic effect of such drugs that has led to moderate success. The studies in children are not randomized, placebo-controlled trials as they were in DUET- ${ }^{5}$ and DUET- $2,{ }^{6}$ which can make the effect of ETR on outcomes difficult to separate. In one study performed primarily in adults in resource-limited settings, patients with NNRTI resistance and NRTI exposure who were PI-naïve were randomized in an open-label trial to receive either ETR or an investigator-selected PI-based regimen in combination with two NRTIs to which they were susceptible on genotype testing. The trial was halted early by the sponsor after an interim analysis showed that those receiving ETR had suboptimal virologic responses compared with those on the PI-based regimen. ${ }^{34}$ It appeared from this study that ETR plus NRTIs alone may not be optimal for salvage of initial NNRTI-based regimen failures, at least in a resource-limited setting. In a cross-sectional study from Mozambique of children treated with at least 6 months of zidovudine or stavudine plus lamivudine plus NVP, 135 (27\%) had VL assays $>50$ copies/mL. Of those, there were 84 samples on which genotypic testing was performed, which revealed the percentage of children infected by virus conferring complete resistance to NVP and EFV was $92 \% .{ }^{35}$ Surprisingly, $6 \%$ of the children 
had ETR resistance with three or more ETR RAMs, despite never having taken the drug. On multivariate analysis, the duration of treatment was the only factor associated with an extended resistance profile, and treatment $>24$ months was of the highest risk. ${ }^{35}$ This high level of NNRTI resistance could be partially explained by the fact that VL monitoring and resistance testing is not a routine part of HIV clinical care in these settings, and first-line NNRTI regimen failures are not captured early prior to the accumulation of multiple ETR RAMs. However, these concerns about the efficacy of ETR in heavily NNRTI-treated pediatric patients also exist in developed settings. Aulicino et $\mathrm{al}^{36}$ evaluated 71 perinatally infected children and adolescents in Argentina who were failing their current regimen with VL assays and resistance testing between the years of 2006 and 2008. By using the ETR resistance score developed by Vingerhoets et al, ${ }^{37} 2.8 \%$ of their patients had a predicted reduced response to ETR (score of $\geq 4$ ), and $19.7 \%$ had a predicted intermediate response to the drug. Given the factors outlined earlier that contribute to the development of resistance, this level of cross-resistance to ETR is likely a universal phenomenon in pediatric HIV clinics across the world, even in resource-rich settings, which could limit its clinical utility in many patients. In the PIANO study, in contrast to the DUET studies in adults, $25 \%$ of the children had never been treated with NNRTIs prior to enrollment, and they could represent up to one-quarter of the $56 \%$ of children who become undetectable on an ETR-containing regimen. ${ }^{25}$ This may indicate that when the drug is given to those children with NNRTI resistance, it could have a much lower rate of virologic suppression than was represented in this study.

\section{Practical role for etravirine in current clinical practice}

Based on the current available data, ETR appears to be best used as part of a third-line salvage cART regimen in the presence of other potent active agents, possibly DRV/r and/ or RAL, in patients with some NNRTI resistance. For this to be possible, providers have to monitor patients closely for VF on regimens containing NVP and EFV, send genotype assays when indicated, and make regimen changes more quickly than in the past. Ongoing active viral replication in the face of these drugs will not only lead to resistance to first-generation NNRTIs (and other ARVs) but may lead to the accumulation of ETR RAMs that will significantly decrease the efficacy of ETR in the future. As more data accumulate regarding the safety, dosing, and efficacy of this drug in younger children, it may also be valuable in treating infants with vertically transmitted NNRTI resistance mutations (especially those who acquire the EFV K103N mutation) or young infants with early NNRTI failure on NVP.

In the future, there may be a role for ETR as first-line treatment in ARV-naïve adults and children. Based on data from the Study of Etravirine Neuropsychiatric Symptoms versus Efavirenz (SENSE) trial in ARV treatment-naïve adults, ETR appeared to be virologically noninferior to EFV when both were combined with two NRTIs, although the study was not powered to make this determination, as it was not the planned initial primary outcome. ${ }^{38}$ Neuropsychiatric events were statistically lower in the ETR arm versus the EFV arm, as would be predicted from their known side effect profiles. In addition, as opposed to adults who had VF on EFV in the trial, those who failed ETR in the study did not fail with any new treatment-emergent NNRTI mutations. ETR was given once daily in this study, as opposed to the currently approved twice-daily dosing scheme, and appeared to retain good activity. Further pharmacokinetic data in children are needed before once-daily dosing can be advocated. There are currently ten ETR clinical trials registered on the NIH clinical trials website involving children or adolescents evaluating pharmacokinetics, use in third-line salvage regimens, or use in combination with other newer agents. ${ }^{39}$

\section{Conclusion}

ETR is now a US Food and Drug Administration-approved option for use in the treatment of pediatric HIV infection with emerging clinical data to support its use. More data in the near future should be available to help guide clinicians in finding the preferred niche for ETR in pediatric HIV treatment.

\section{Disclosure}

The author reports no conflicts of interest in this work.

\section{References}

1. Rogers MF, Thomas PA, Starcher ET, Noa MC, Bush TJ, Jaffe HW Acquired immunodeficiency syndrome in children: report of the Centers for Disease Control National Surveillance, 1982 to 1985. Pediatrics. 1987;79(6):1008-1014.

2. Krasinski K, Borkowsky W, Holzman RS. Prognosis of human immunodeficiency virus infection in children and adolescents. Pediatr Infect Dis J. 1989;8(4):216-220.

3. Patel K, Hernan MA, Williams PL, et al. Long-term effectiveness of highly active antiretroviral therapy on the survival of children and adolescents with HIV infection: a 10-year follow-up study. Clin Infect Dis. 2008;46(4):507-515.

4. Panel on Antiretroviral Therapy and Medical Management of HIVinfected Children. Guidelines for the use of antiretroviral agents in pediatric HIV infection. http://aidsinfo.nih.gov/contentfiles/lvguidelines/ pediatricguidelines.pdf. Accessed March 11, 2013. 
5. Madruga JV, Cahn P, Grinsztejn B, et al. Efficacy and safety of TMC125 (etravirine) in treatment-experienced HIV-1-infected patients in DUET-1: 24-week results from a randomised, double-blind, placebocontrolled trial. Lancet. 2007;370(9581):29-38.

6. Lazzarin A, Campbell T, Clotet B, et al. Efficacy and safety of TMC125 (etravirine) in treatment-experienced HIV-1-infected patients in DUET-2: 24-week results from a randomised, double-blind, placebocontrolled trial. Lancet. 2007;370(9581):39-48.

7. Katlama C, Haubrich R, Lalezari J, et al. Efficacy and safety of etravirine in treatment-experienced, HIV-1 patients: pooled 48 week analysis of two randomized, controlled trials. Aids. 2009;23(17):2289-2300.

8. Tibotec Pharmaceuticals Inc. INTELENCE (etravirine) tablets for oral use: US prescribing information. http://www.intelence.com/sites/ default/files/pdf/INTELENCE_Booklet_Package_Insert_hcp.pdf. Accessed March 11, 2013.

9. Shearer WT, Quinn TC, LaRussa P, et al. Viral load and disease progression in infants infected with human immunodeficiency virus type 1. N Engl J Med. 1997;336(19):1337-1342.

10. Walker AS, Doerholt K, Sharland M, Gibb DM, Collaborative HIVPSSC. Response to highly active antiretroviral therapy varies with age: the UK and Ireland Collaborative HIV Paediatric Study. Aids. 2004;18(14):1915-1924.

11. Chadwick EG, Capparelli EV, Yogev R, et al. Pharmacokinetics, safety and efficacy of lopinavir/ritonavir in infants less than 6 months of age: 24 week results. Aids. 2008;22(2):249-255.

12. Little SJ, Holte S, Routy JP, et al. Antiretroviral-drug resistance among patients recently infected with HIV. $N$ Engl J Med. 2002; 347(6):385-394.

13. Johnson VA, Petropoulos CJ, Woods CR, et al. Vertical transmission of multidrug-resistant human immunodeficiency virus type 1 (HIV-1) and continued evolution of drug resistance in an HIV-1-infected infant. J Infect Dis. 2001;183(11):1688-1693.

14. Cecchini DM, Zapiola I, Fernandez Giuliano S, Martinez MG, Rodriguez CG, Bouzas MB. Etravirine resistance mutations in HIVinfected pregnant women. HIV Med. 2013;14(2):125-126.

15. Musiime V, Ssali F, Kayiwa J, et al. Response to nonnucleoside reverse transcriptase inhibitor-based therapy in HIV-infected children with perinatal exposure to single-dose nevirapine. AIDS Res Hum Retroviruses. 2009;25(10):989-996.

16. Palumbo P, Lindsey JC, Hughes MD, et al. Antiretroviral treatment for children with peripartum nevirapine exposure. $N$ Engl J Med. 2010; 363(16):1510-1520.

17. Gibb DM, Goodall RL, Giacomet V, Mcgee L, Compagnucci A, Lyall H. Adherence to prescribed antiretroviral therapy in human immunodeficiency virus-infected children in the PENTA 5 trial. Pediatr Infect Dis J. 2003;22(1):56-62.

18. Bikaako-Kajura W, Luyirika E, Purcell DW, et al. Disclosure of HIV status and adherence to daily drug regimens among HIV-infected children in Uganda. AIDS Behav. 2006;10:85-93.

19. de Mulder M, Yebra G, Navas A, et al. High drug resistance prevalence among vertically HIV-infected patients transferred from pediatric care to adult units in Spain. PloS One. 2012;7(12):e52155.

20. Delaugerre C, Rohban R, Simon A, et al. Resistance profile and cross-resistance of HIV-1 among patients failing a non-nucleoside reverse transcriptase inhibitor-containing regimen. $J$ Med Virol. 2001;65(3):445-448.

21. Vingerhoets J, Azijn H, Fransen E, et al. TMC125 displays a high genetic barrier to the development of resistance: evidence from in vitro selection experiments. J Virol. 2005;79(20):12773-12782.

22. Vingerhoets J, Tambuyzer L, Azijn H, et al. Resistance profile of etravirine: combined analysis of baseline genotypic and phenotypic data from the randomized, controlled Phase III clinical studies. Aids. 2010;24(4):503-514.
23. Konigs C, Feiterna-Sperling C, Esposito S, et al. Pharmacokinetics and short-term safety and tolerability of etravirine in treatment-experienced HIV-1-infected children and adolescents. Aids. 2012;26(4):447-455.

24. Tibotec Pharmaceuticals Inc. TMC125-TiDP35-C213: Safety and antiviral activity of etravirine (tmc125) in treatment-experienced, HIV infected children and adolescents. http://clinicaltrials.gov/ct2/show/ NCT00665847. Accessed March 11, 2013.

25. Tudor-Willaims G, Cahn P, Chokephaibulkit K, et al. Safety and efficacy of etravirine in HIV-1-infected, treatment-experienced children and adolescents: PIANO 48-week results. AIDS 2012: XIX International AIDS Conference. Washington, DC, July 22-27, 2012.

26. US Food and Drug Administration. Intelence (etravirine): pediatric dosing recommendations and new scored $25 \mathrm{mg}$ tablet for pediatric dosing. http://www.fda.gov/ForConsumers/ByAudience/ForPatientAdvocates/ HIVandAIDSActivities/ucm297471.htm. Accessed March 11, 2013.

27. Janssen R\&D Ireland. TMC125-TiDP35-C239- Continued access to etravirine (ETR) in treatment experienced HIV-1 infected children and adolescents. http://www.clinicaltrials.gov/ct2/show/study/NCT009805 38?term=etravirine\&rank=13. Accessed March 11, 2013.

28. National Institute of Allergy and Infectious Diseases (NIAID). Evaluating the safety and tolerability of etravirine in HIV-1 infected infants and children. http://www.clinicaltrials.gov/ct2/show/study/NCT01504841? term=children+etravirine\&rank=1. Accessed March 11, 2013.

29. Ananworanich J, Prasitsuebsai W, Kosalaraksa P, Kanjanavanit S, Ngampiyaskul C, Wongsawat J. Outcomes of third-line antiretroviral therapy containing darunavir, etravirine or raltegravir in Thai children with HIV infection. XIX International AIDS Conference; Washington, DC, July 22-27, 2012.

30. Thuret I, Chaix ML, Tamalet C, et al. Raltegravir, etravirine and r-darunavir combination in adolescents with multidrug-resistant virus. Aids. 2009;23(17):2364-2366.

31. Briz V, Palladino C, Navarro M, et al. Etravirine-based highly active antiretroviral therapy in HIV-1-infected paediatric patients. HIV Med. 2011;12(7):442-446.

32. Vigano A, Meroni L, Marchetti G, et al. Successful rescue therapy with a darunavir/ritonavir and etravirine antiretroviral regimen in a child with vertically acquired multidrug-resistant HIV-1. Antivir Ther. 2008;13(6):839-843.

33. Johnson VA, Calvez V, Gunthard HF, et al. 2011 update of the drug resistance mutations in HIV-1. Top Antivir Med. 2011;19(4):156-164.

34. Ruxrungtham K, Pedro R, Latiff G, et al. Impact of reverse transcriptase resistance on the efficacy of TMC125 (etravirine) with two nucleoside reverse transcriptase inhibitors in protease inhibitor naïve, nonnucleoside reverse transcriptase inhibitor-experienced patients: study TMC125-C227*. HIV Med. 2008;9(10):883-896.

35. Vaz P, Chaix ML, Jani I, et al. Risk of extended viral resistance in human immunodeficiency virus-1-infected Mozambican children after first-line treatment failure. Pediatr Infect Dis J. 2009;28(12):e283-e287.

36. Aulicino PC, Rocco CA, Mecikovsky D, Bologna R, Mangano A, Sen L. HIV type-1 genotypic resistance profiles in vertically infected patients from Argentina reveal an association between K103N+L100I and L74V mutations. Antivir Ther. 2010;15(4):641-650.

37. Vingerhoets J, Peeters M, Azijn H, et al. An update of the list of NNRTI mutations associated with decreased virological response to etravirine: multivariate analyses on the pooled DUET-1 and DUET-2 clinical trial data. Antivir Ther. 2008;13(Suppl 3):A26.

38. Gazzard B, Duvivier C, Zagler C, et al. Phase 2 double-blind, randomized trial of etravirine versus efavirenz in treatment-naive patients: 48-week results. Aids. 2011;25(18):2249.

39. US National Institues of Health. http://www.clinicaltrials.gov/ct2/resu lts?term=etravirine+children. Accessed March 11, 2013. 
HIV/AIDS - Research and Palliative Care

Dovepress

\section{Publish your work in this journal}

HIV/AIDS - Research and Palliative Care is an international, peerreviewed open-access journal focusing on advances in research in HIV, its clinical progression and management options including antivira treatment, palliative care and public healthcare policies to control viral spread. The journal welcomes original research, basic science, clinical \& epidemiological studies, reviews \& evaluations, expert opinion \& commentary, case reports \& extended reports. The manuscript management system is completely online and includes a very quick and fair peer-review system. Visit http://www.dovepress.com/ testimonials.php to read real quotes from published authors.

Submit your manuscript here: http://www.dovepress.com/hivaids---research-and-palliative-care-journal 